Mens

revue d'histoire intellectuelle de l'Amérique française

Yvan Lamonde et Sophie Montreuil, dir. Lire au Québec au XIX ${ }^{e}$ siècle. Montréal, Fides, 2003.330 p.

\title{
Francis Parmentier
}

Volume 5, numéro 2, printemps 2005

URI : https://id.erudit.org/iderudit/1024371ar

DOI : https://doi.org/10.7202/1024371ar

Aller au sommaire du numéro

Éditeur(s)

Centre de recherche en civilisation canadienne-française

ISSN

1492-8647 (imprimé)

1927-9299 (numérique)

Découvrir la revue

Citer ce compte rendu

Parmentier, F. (2005). Compte rendu de [Yvan Lamonde et Sophie Montreuil, dir. Lire au Québec au XIX ${ }^{e}$ siècle. Montréal, Fides, 2003. 330 p.] Mens, 5(2),

537-556. https://doi.org/10.7202/1024371ar d'utilisation que vous pouvez consulter en ligne.

https://apropos.erudit.org/fr/usagers/politique-dutilisation/ 
ment justifier l'inclusion de ce dernier problème. Dans ce cheminement, le lecteur a du mal à saisir l'évolution et la fécondité de la comparaison. En fait, les liens entre les chapitres sont déficients ou inexistants. Heureusement, Jacques Prévotat parvient, en conclusion, à donner un certain sens à cet ouvrage en complétant et en redressant parfois, sans y paraître, les propos de Claude Hauser et de Catherine Pomeyrols dans l'introduction. On est frappé de constater que cet ouvrage ne présente aucune orientation bibliographique. Ce fait est particulièrement étonnant lorsque l'on sait que l'un des objectifs des auteurs est d'attirer les chercheurs vers ces questions. Dans cette optique, on aurait aimé être renseigné sur l'état actuel des recherches. Le travail d'édition a été bâclé. La présentation biographique des collaborateurs en oublie deux sur huit. Le contenu de la note 64 (p. 114) a été reporté au texte (p. 104), sans qu'on pense à la supprimer. Oublions les coquilles. Néanmoins, malgré ces nombreuses lacunes, cet ouvrage introduit des pistes de réflexion sur un sujet méconnu et peu exploité.

Stéphane St-Pierre

Département d'bistoire

Université de Montréal

\section{Yvan Lamonde et Sophie Montreuil, dir. Lire au Québec au $\boldsymbol{X I X}^{\mathrm{e}}$ siècle. Montréal, Fides, 2003. 330 \\ p.}

Tous ceux que passionne l'histoire culturelle se réjouiront de la sortie de cet ouvrage. L'histoire du livre et de la lecture, dont Yvan Lamonde a été un des éminents pionniers au Québec, se trouve ici enrichie d'une série d'études classées en deux parties dans le présent ouvrage: "Pratiques in- 
dividuelles de lecture et littérature personnelle », et "Pratiques individuelles et collectives de lecture à travers l'étude de catalogues ». Dans un chapitre préliminaire intitulé « Pour une histoire des pratiques de lecture : éléments de méthode et pacte fondateur ", Lamonde et Montreuil rappellent que «[1]'histoire de la lecture est la dernière-née du domaine plus général de l'histoire du livre et de l'édition, tant sur le plan québécois qu'international (p. 8).» Les collaborateurs de ce dernier-né tenteront donc une percée tant au plan épistémologique qu'au plan des connaissances, en s'attardant aux pratiques de lecture individuelles et collectives, se fondant soit sur des documents qui relèvent du domaine de l'intime (correspondances, journaux intimes), soit du domaine public (catalogues d'institutions scolaires ou de bibliothèques privées ou publiques).

Disons d'entrée de jeu qu'une matière aussi abondante défie le résumé ou la synthèse, aussi procéderons-nous de manière quelque peu scolaire en tentant de dégager les éléments les plus saillants de chacun des huit chapitres.

Le premier, «Lectures domestiques, d'exil et de retraite de Louis-Joseph Papineau (1823-1871) », par Yvan Lamonde et Frédéric Hardel, s'appuie sur la correspondance du grand homme pour en tirer un portrait du lecteur, et ceci dans tous ses aspects : le collectionneur de livres, ses auteurs préférés, ses habitudes de lecture, ses lieux de lecture. Le profil qui s'en dégage me semble être le mieux résumé par Papineau luimême quand il confie à son fils Amédée en 1857: «Ma solitude et ma bibliothèque sont donc pour moi le meilleur séjour possible (p. 19).» Et je me suis demandé si Papineau ne rejoint pas ici Montaigne, comme lui homme d'action, qui se réfugiera dans sa tour, et comme Papineau lui-même en 1856 dans une tour qu'il fait construire à quelques mètres de sa maison de Montebello. Il est vrai que, comme le font remar- 
quer à juste titre les auteurs du chapitre, un rapprochement avec Voltaire s'avérerait tout aussi pertinent, le Voltaire du Candide en particulier, Papineau combinant l'amour des livres et de l'horticulture.

Comme tous les hommes cultivés de son temps, Papineau voue un véritable culte aux Anciens, et tout particulièrement à Sénèque, le moraliste, qu'il avoue aimer et admirer " plus qu'aucun autre (p. 40) », ainsi qu'à Cicéron, modèle du tribun et « un auteur avec lequel on ne peut jamais assez se familiariser (p. 34). » Après Sénèque et Cicéron, c'est à Voltaire que va son admiration car " [il] a plus fait pour abattre l'esprit d'intolérance et de persécution religieuse que nul autre homme (p. 42). " L'on comprend par ailleurs son admiration pour Lamennais et sa tentative de concilier démocratie et christianisme, ainsi que pour Tocqueville dont la lecture de la Démocratie en Amérique ne pouvait qu'accentuer sa profonde admiration pour la République américaine.

Il est clair que la lecture des journaux occupe une partie de son temps - à son corps défendant d'ailleurs car il écrit : "Une lecture hâtive de journaux pour être au courant de ce qui se passe est nécessaire, mais, pour être instruit, juste et sage, il faut bien peu de journaux et beaucoup de bons livres [...] Le journalisme seul ne fera jamais que des ignorants passionnés (p. 59)» — car elle seule permet au citoyen de suivre le cours des événements et de se former une opinion. Au cours de son exil, il a pu se familiariser avec la presse américaine, qu'il admire, mais dont il n’ignore pas les faiblesses : «Sous le rapport de la hardiesse à mentir et à écrire grossièrement, les journaux américains sont les plus intolérables de tous (p. 59).» La presse française n'échappe pas plus à sa critique : «la presse française est trop dirigée dans un esprit de parti et trop peu consciencieuse (p. 57). " ̀̀ Montebello, il lit la plupart des journaux canadiens, anglais et français, dont L'Avenir, rem- 
placé bientôt par Le Pays, dont il est d'ailleurs un des bailleurs de fonds. Quand ce dernier fait campagne en faveur de l'abolition du régime seigneurial - à laquelle le Seigneur de Montebello est naturellement opposé - il déplore qu'« il n'y a nul autre journalisme au monde tombé aussi bas que l'est le journalisme canadien (p. 62). »

On a déjà dit que le lieu de lecture favori de Papineau était sa "tour", après sa construction, naturellement, mais il aime aussi lire au lit. D'autre part, la lecture se fait souvent en famille, activité qui resserre les liens de la cellule familiale et donne tôt aux jeunes le goût de la lecture.

L'intérêt de ce chapitre est double: d'une part il nous renseigne sur les habitudes de lecture d'un des personnages les plus influents du $\mathrm{XIX}^{\mathrm{e}}$ siècle québécois, et d'autre part il nous fait mieux connaittre l'homme, aussi bien public que privé. Papineau est bien ce libre-penseur, un esprit « fort » comme on disait alors avec un brin d'ironie, qui voyait dans les ÉtatsUnis le modèle sur lequel les Québécois devraient s'aligner.

Le chapitre deux s'intitule : « La lecture et "le livre de l'histoire " chez Amédée Papineau (1835-1845) ", par Yvan Lamonde. Le corpus étudié est plus diversifié que dans le chapitre précédent : « journal personnel, souvenirs, abondante correspondance expédiée et reçue, bref catalogue de sa bibliothèque de 1841 (p. 69). » Il est clair que les lectures du fils seront orientées principalement vers la connaissance et l'interprétation des événements politiques de l'époque. Or, il est intéressant de remarquer qu'Amédée apprendra très tôt à lire couramment en anglais et en français, le bilinguisme étant couramment pratiqué dans l'élite canadienne-française. Amédée sera lui aussi un avide lecteur de journaux, notamment du Canadien de Parent, en même temps qu'un admirateur, comme son père, de Lamennais et de Tocqueville. Il note dans son Journal d'un Fils de la Liberté: "Je viens de terminer la lecture 
de l'admirable, du délicieux ouvrage de Tocqueville, De la démocratie en Amérique. Il occupera toujours une place favorite et saillante sur les rayons de ma bibliothèque. Je le relirai bien des fois, je l'étudierai souvent. Paine, Lamennais, Tocqueville, trinité démocratique que je révère (p. 79)!» En littérature, en homme de son temps, Amédée affectionne particulièrement le roman historique qui l'aide à mieux comprendre son époque.

Amédée est né dans un milieu où non seulement les livres abondent, mais où ils sont révérés. De plus, non seulement la famille Papineau entretient-elle des liens étroits avec les libraires Bossange et Fabre, mais Amédée a accès aux bibliothèques privées d'amis de la famille. De plus, au cours de ses séjours à l'étranger, il se familiarisera avec les grandes bibliothèques publiques américaines et européennes qui font encore cruellement défaut au Canada.

Amédée est un lecteur boulimique, les jeux de société l'intéressant fort peu, et l'on se plaît à rêver à ce que pouvait être alors " [la] soirée passée au salon près d'un bon feu, à lire les ouvrages si amusants de Dickens (p. 88). "Sa curiosité intellectuelle ne semble pas connaître de limites, même si la politique vient en premier. Ce chapitre trace ainsi le portrait d'un des premiers grands intellectuels québécois.

Dans «Lectures de Lactance Papineau, un "Canadien malheureux" ", Frédéric Hardel étudie les habitudes de lecture du frère d'Amédée, qui deviendra médecin pour faire plaisir à son père, et finira ses jours, victime de la maladie mentale, dans une institution religieuse française à Lyon. Lactance, comme son père et son frère, est lui aussi un homme du livre, et même si ses études médicales l'obligent à se consacrer à des lectures scientifiques, il n'en délaisse pas pour autant la littérature si l'on en juge par la composition de sa bibliothèque, dont le catalogue révèle que « [la] section médicale est à 
peine plus importante que la section littéraire (p. 115). » Aux auteurs gréco-latins, dont Sénèque, s'ajoutent les grands auteurs français du XVI ${ }^{e}$ au XIX ${ }^{e}$, jusqu'à Lamartine et Chateaubriand, et quelques écrivains allemands, italiens, ainsi que les romans historiques de Walter Scott et Les Mille et une Nuits.

Frédéric Hardel se base sur la correspondance, le journal de Lactance et le catalogue de la correspondance reconstitué pour peindre le portrait du lecteur qu'a été Lactance. Même si la documentation est ici plus lacunaire, il n'en demeure pas moins que nous pouvons suivre le jeune homme du Séminaire de Saint-Hyacinthe, où il devient membre de la Société littéraire fondée par Amédée, et s'adonne semble-t-il essentiellement à la lecture des journaux - La Minerve, L'Écho du pays, le Herald - jusqu'à Paris, où il étudie la médecine et se monte une bibliothèque composée essentiellement d'ouvrages de sa discipline, mais aussi d'ouvrages à caractère littéraire. Il lit avec ivresse, à la fois par devoir et par plaisir, passant d'un traité sur l'onanisme au René et Atala de Chateaubriand, les Mystères de Paris que lui a prêté le libraire Bossange, et «les leçons de MM. Michelet et Quinet contre les Jésuites (p. 111).»

Un aspect de la «carrière » du lecteur Lactance à Paris qui mérite d'être souligné, son aspect «mondain » si l'on préfère, c'est à la fois la fréquentation du théâtre, et son appartenance à ce qu'on qualifierait de nos jours de cercle de lecture, les Compagnons d'Isis, "à prétentions littéraires, philosophiques et artistiques (p. 113)", dont le fondateur fut nul autre que le comte de Gobineau!

Ces trois premiers chapitres ne prétendent pas donner de la lecture au XIX siècle au Québec un aperçu représentatif. Non seulement Louis-Joseph Papineau est-il un personnage hors du commun, mais ses deux fils ont eux aussi vécu l'exil aux États-Unis et en France, un exil qui leur fut bénéfi- 
que à plus d'un titre, notamment au chapitre de la culture livresque. Néanmoins, il est permis de penser que d'autres représentants de l'élite francophone de leur temps cultivaient eux aussi la lecture, comme le chapitre suivant, " $(\mathrm{Se})$ lire et (se) dire : Joséphine Marchand-Dandurand et la lecture (18791886) », l'illustre bien.

Évidemment, nous abordons ici une tout autre époque que celle décrite précédemment, un milieu social et une idéologie qui, tout en ayant des points communs, sont cependant éloignés l'un de l'autre. Et bien entendu, le personnage est féminin. Les sources sont ici le journal intime et une abondante correspondance (981 lettres) avec son mari, le sénateur Dandurand. Rappelons que Joséphine a été « [auteure] de chroniques destinées aux journaux, de contes et de pièces de théâtre, engagée dans la cause des femmes et auprès d'œuvres charitables (p. 123). » Il s'agit donc bien d'une femme originaire d'un milieu privilégié, économiquement et culturellement parlant, intelligente, énergique, et décidée à contribuer au mieux-être de ses concitoyennes puisqu'elle est notamment fondatrice d'une organisation philanthropique " qui avait pour mission d'expédier des livres gratuitement à des institutrices localisées aux quatre coins du Québec (et audelà des frontières québécoises) (p. 123). " Précisons toutefois qu'il s'agira ici du portrait de la jeune lectrice.

On ne sera guère choqué - peut-être amusé - d'apprendre que la censure est omniprésente. Ce sont tantôt la mère, tantôt le futur mari, tantôt le confesseur qui veillent au grain, i.e. à l'édification morale d'une jeune fille de bonne famille! Comme le confie cette jeune fille pas comme les autres : "L'inspection maternelle dresse entre ma trouvaille et moi son lorgnon vigilant (p. 134). » La plupart des titres mentionnés relèvent de la littérature «bon ton» de l'époque, mais de temps en temps se glissent des ouvrages qui risquent 
de troubler les sens d'une jeune provinciale. Telle est l'interprétation, du moins, qu'en donne la mère qui consent finalement, après vérification rapide, à lui laisser lire L'bistoire d'une Parisienne d'Octave Feuillet. Malgré "sa moue incertaine", elle est convaincue néanmoins que «cela peut avoir son utilité (p. 134).»

Joséphine sait faire preuve d'indépendance d'esprit et il lui arrive d'outrepasser les interdits maternels, ou de refuser de s'en référer à son confesseur, de peur de connaitre la réponse! Elle ne risque pas cependant de devenir une autre Emma Bovary, elle qui a reçu une excellente éducation religieuse et qui a lu La femme forte: Conférences destinées aux femmes du monde d'un certain $\mathrm{M}^{\mathrm{gr}}$ Landriot, ou deux chapitres de L'imitation de Jésus-Cbrist en compagnie de son mari «après avoir été à la messe comme de bons chrétiens (p. 137). »

On ne sera pas surpris d'apprendre que les auteurs français ont sa préférence, à commencer par les classiques et les romantiques - certains d'entre eux, tout au moins - et quelques auteurs contemporains auxquels on a fait allusion plus haut. Naturellement, elle se dit " révoltée » par Zola, Gautier et Daudet, mais on reste perplexe face à la présence de La vie de Jésus-Christ [sic] de Renan dans sa bibliothèque!

Ici encore, comme chez les Papineau, on retrouve le rôle central de la lecture des journaux, souvent faite à haute voix et en famille. La carrière littéraire et politique de son père, puis la carrière politique de son mari, ne sont bien entendu pas étrangères à l'intérêt que prend la jeune femme aux journaux: "Je reçois tous les jours La Patrie et le Star. Vous êtes bien mignon. Ça me fait un gros plaisir (p. 136). ", écritelle à Raoul en 1885.

Les futurs époux partagent une passion égale pour la lecture et comme le dit Sophie Montreuil : «Des mots pour s'écrire, des mots pour écrire : la lecture n'est pas qu'un inter- 
médiaire pour se comprendre, elle est la matière première qui nourrit la plume de Joséphine et de Raoul (p. 145). »

Ce chapitre nous fait donc pénétrer dans l'intimité d'une jeune fille intelligente et cultivée de bonne famille libérale au XIX ${ }^{\mathrm{e}}$. Il y en eut d'autres, certainement, mais elles n'ont point laissé de traces. Aujourd'hui, grâce à l'accès aux études supérieures, plus de jeunes filles qu'alors lisent, mais sans confesseur ni censure maternelle...

Nous abordons maintenant la deuxième partie de l'ouvrage intitulée "Pratiques individuelles et collectives de lecture à travers l'étude de catalogues », qu'ouvre le chapitre "Les catalogues et leur contribution à une histoire des pratiques de lecture au Québec ", d'Andrea Rotundo. À vrai dire, il s'agit plutôt d'une brève introduction rappelant l'existence de la «base de données des Catalogues canadiens relatifs à l'imprimé / Canadian Book Catalogues (CCRI/CBC), créée dans le cadre du projet "History of the Book in Canada / Histoire du livre et de l'imprimé au Canada" et comportant plus de mille entrées, [qui] permet l'analyse systématique des différents types de catalogues produits et distribués au Canada pendant environ 200 ans, soit depuis les années 1750 jusqu'au milieu du XXe siècle (pp. 153-154). » Les chercheurs et les amateurs de l'histoire du livre et de la lecture ne peuvent que se réjouir de l'existence d'une telle base de données qui enrichit notre connaissance de l'histoire culturelle du Québec et de ses pratiques de lecture.

Puis nous entrons dans le vif du sujet avec «Voyage autour d'une bibliothèque : la littérature dans les catalogues de bibliothèques personnelles d'écrivains (1880-1910)", de Annie-Claude Prud'homme. Sont analysés dans le détail les catalogues d'Honoré Beaugrand, Oscar Dunn, Joseph Doutre, Pamphile Lemay, Louis-Wilfrid Marchand, Faucher de SaintMaurice et Jules Fournier. De ce vaste corpus se dégagent les 
caractéristiques suivantes : (a) Comme nous l'avons déjà constaté aux chapitres précédents, la place du journalisme est considérable dans toutes ces bibliothèques, et elle se manifeste notamment par la présence des pamphlets, des chroniques, et des feuilletonistes, tels Alexandre Dumas, Eugène Sue et Frédéric Soulié ; (b) La littérature "nationale » s'impose de plus en plus puisque au-delà de $42 \%$ des titres littéraires sont québécois. Le roman d'ici, sans être prioritaire, fait néanmoins une percée intéressante; (c) La lecture s'alimente aux sources européennes, surtout françaises, américaines et québécoises ; (d) Les préromantiques et romantiques français - Bernardin de Saint-Pierre et Chateaubriand - prennent dorénavant le pas sur les classiques.

Nul doute que l'examen attentif des catalogues des bibliothèques d'écrivains met les lecteurs de ces derniers sur des pistes intéressantes quant aux influences littéraires et/ou idéologiques qu'ils ont subies.

1) Honoré Beaugrand (1848-1906), qui fut romancier, journaliste, essayiste et politicien, monta une bibliothèque riche et diversifiée. Esprit libre, volontiers iconoclaste, il ne s'embarrasse guère des directives du clergé, aussi s'entoure-til d'ouvrages classés à l'Index, tels : La Lanterne d'Arthur Buies, des œuvres de Zola, les œuvres complètes de Voltaire, et des pamphlets anticléricaux de Léo Taxil : $\grave{A}$ bas la calotte et Les friponneries religieuses. Les romans constituent, comme il se doit, la partie la plus importante de la collection, avec 33,6\% des titres, dont seulement neuf titres québécois. Amateur d'opéra - 33 titres - et de littérature de langue anglaise - il a vécu, comme l'on sait, aux États-Unis - il affectionne plus particulièrement les " œuvres complètes ", dont celles de Dickens, Scott, Rousseau, Voltaire, Hugo, etc. En tout, le catalogue de sa bibliothèque recense 729 titres et 921 volumes. 
2) Oscar Dunn (1845-1885). Deux catalogues, le premier de 1880 et le second de 1885, ont été publiés, révélant dans le premier cas une proportion de $20,6 \%$ de littérature, dont majoritairement de la poésie, tandis que cette proportion passe à 40,8\% en 1885, avec une majorité consacrée au roman. Signalons une forte proportion - 35\% - de titres canadiens dans la catégorie " ouvrages linguistiques » et un nombre respectable de titres canadiens - 11 sur 31 - dans la catégorie "histoire et critique littéraires ». Dunn se révèle un fin lettré, à tout le moins si l'on en juge par un pourcentage élevé d'ouvrages d'auteurs grecs et latins, et la présence des grands de la littérature universelle: Dante, Cervantès, Shakespeare. Dunn, on le sait, est de tendance conservatrice, défenseur des valeurs traditionnelles, religion, patrie, etc.

3) Joseph Doutre (1825-1886), à l'inverse, fut la bête noire du clergé, ardent collaborateur et défenseur de l'Institut canadien. Le catalogue de l'auteur des Fiancés de 1812 ne compte guère que 250 titres pour 700 volumes. La majorité des titres porte sur le droit - rappelons qu'il fut l'avocat de la veuve Guibord - et une faible minorité sur la littérature : $5,2 \%$.

4) Pamphile Lemay (1837-1918). Lemay, poète, traducteur et bibliothécaire, vénère la littérature à laquelle il assigne une mission patriotique. Aussi ne s'étonnera-t-on pas de trouver dans son catalogue de 861 volumes une proportion de $44,9 \%$ d'œuvres littéraires, dont une proportion élevée est québécoise : 41,9\%. Il affectionne particulièrement les romans de cape et d'épée et, bien entendu, la poésie.

5) Louis-Wilfrid Marchand (1834-1896), greffier des appels à Montréal, est donc un de ces anonymes amateurs de livres, dont le catalogue recense une proportion égale d'ouvrages de droit et de littérature, ces derniers regroupant beau- 
coup de feuilletonistes français, mais aussi Homère et Virgile, Molière, Racine et Corneille. Ici, l'éclectisme domine.

6) Faucher de Saint-Maurice (1844-1897). Auteur de nombreux récits de voyage, journaliste, député, vice-président de la section française de la Société royale, il n'est guère surprenant que sa bibliothèque regorge de récits de voyage sur les Amériques, de périodiques, surtout québécois, ainsi que d'ouvrages de critique littéraire dont la majorité sont québécois eux aussi.

7) Jules Fournier (1884-1918). Journaliste, pamphlétaire que ses idées envoyèrent en prison, il publia lui-même un catalogue de sa bibliothèque à l'intention de ses amis intitulé La Cité des Livres, dans lequel il «classe les volumes de sa bibliothèque en diverses sections numériques (p. 185). » On notera l'importance des auteurs français, notamment de mécréants comme Anatole France ; de Jules Lemaitre, « qui semble avoir influencé le plus Jules Fournier et par les idées et par le style (p. 185).» Mais la section «Canadiana » n'est point négligeable puisqu'elle occupe 19,3\% de l'ensemble. Pamphlétaire, il s'inspire de Léon Bloy, Rochefort, Veuillot et Buies.

En terminant, Annie-Claude Prud'homme rappelle qu'il faut être prudent quant aux conclusions que l'on tirera de cette étude des catalogues qui ne sont pas représentatifs de l'ensemble des bibliothèques de leurs propriétaires. Néanmoins, un constat s'impose : la proportion des ouvrages québécois augmente constamment au cours du siècle. Par ailleurs, l'importance des journaux est considérable, ces derniers véhiculant non seulement une bonne partie des romans de l'époque sous forme de feuilletons, mais constituant également le lieu privilégié des grandes batailles idéologiques.

Isabelle Monette, quant à elle, étudie «L'offre de titres littéraires dans les catalogues de la librairie montréalaise (1816-1879) », en l'occurrence cinq catalogues des librairies 
Bossange, Rolland, Fabre et Beauchemin, dont les dates de parution s'échelonnent de 1823 à 1879 . Il est vrai que la librairie du $\mathrm{XIX}^{e}$ prend souvent l'aspect d'un capharnaüm allant du livre aux cartes à jouer, en passant par la chaussure pour dame et le "vrai gruyère ", car « les premiers libraires du Bas-Canada étaient avant tout des commerçants (p. 204).» Ce qu'ils sont demeurés, me semble-t-il...

La librairie Bossange n'est qu'une succursale de la maison parisienne, le fils ayant tenu boutique à Montréal de 1815 à 1819 et, après avoir épousé Julie Fabre, la sœur d'ÉdouardRaymond Fabre, rentre en France pour y ouvrir sa propre librairie. Ce catalogue reflète donc vraisemblablement davantage les goûts d'un jeune Parisien bien- pensant — n'oublions pas que la France est alors en pleine période de Restauration - que ceux d'un jeune Montréalais. Abondent donc les ouvrages à caractère religieux, ou faisant l'apologie du christianisme comme Les martyrs ou le triomphe de la religion cbrétienne de Chateaubriand, mais aussi des ouvrages de littérature ancienne, du siècle classique et, audace suprême, jusqu'aux Lettres persanes de Montesquieu! Parmi les auteurs les plus présents selon le nombre de titres et volumes littéraires, Rousseau apparait en troisième position, mais sans que l'on sache de quels ouvrages il s'agit. Dommage, car Voltaire, lui, est absent de la liste, même si l'on sait que son poème La Henriade figure par ailleurs dans la section poésie.

Le catalogue suivant - celui de la maison Fabre, Édouard-Raymond, le fondateur de la dynastie, qui a fait un stage d'un an à Paris en 1822 à la Librairie Bossange, et racheté le fonds de commerce de l'ancienne librairie Bossange de Montréal en 1823 - constitue l'objet de cette étude. Fait à noter, alors que la poésie dominait le catalogue de 1816, c'est maintenant le roman qui l'emporte et qui, ajoutons-le, n'est pas près de céder sa place. D'ailleurs on retrouve des 
traductions d'écrivains de langue anglaise, tels Swift, Walter Scott - ce dernier très populaire en France, comme en atteste le culte que lui portera Balzac - Goldsmith, Richardson, De Foe. Du côté français, Mme de Genlis, qui occupait le premier rang en 1816, a été distanciée en 1823 et occupe le $3^{\mathrm{e}}$ rang derrière Scott et Pigault-Lebrun. Ce dernier signe des romans licencieux, fort à la mode, et dont la popularité auprès du public canadien semblerait suggérer que le clergé n'a pas encore le pouvoir de censurer qu'il acquerra après 1840 .

Le catalogue de 1830 de la maison Fabre révèle une croissance très nette des œuvres littéraires - plus de $92 \%$, rien de moins - par rapport au catalogue de 1823 , avec $50 \%$ des titres attribués au roman, et $20 \%$ à la poésie. Si Scott se maintient en tête - c'est le best-seller de l'époque - Mme de Genlis a repris du terrain et occupe la deuxième position.

Avec le catalogue de la maison Rolland - datant de 1854 - on entre de plain-pied dans l'ère des manuels de classe et des ouvrages d'édification sortis de l'imprimerie du libraireéditeur, et de la vente de nombreux articles de papeterie. Souvenons-nous que $\mathrm{M}^{\mathrm{gr}}$ Bourget veille au grain et que dès lors « une énorme quantité d'ouvrages religieux occupe la majeure partie du catalogue. La clientèle ecclésiastique et les relations commerciales qu'il tente d'établir avec elle sont très précieuses pour Rolland (p. 211). » D'ailleurs, le catalogue mentionne prudemment que la maison se prévaut des conseils d'un ecclésiastique !

On ne s'étonnera donc pas de trouver un nombre considérable d'ouvrages pour la jeunesse issus des collections « $\mathrm{Bi}$ bliothèque morale de la jeunesse » et «Bibliothèque des écoles chrétiennes ». Commerce oblige. Toutefois on retrouve aussi, à l'intention des adultes probablement, les romans de Fenimore Cooper et Walter Scott, Dickens et Hugo, ainsi que les classiques d'usage. 
Le contenu de ce catalogue me semble particulièrement intéressant dans la mesure où il signale un tournant décisif dans l'histoire de la librairie et de l'édition au Québec. Dorénavant, et jusque dans les années 1960, une librairie francophone ne pourra survivre que si elle s'aligne sur les directives de l'évêché et/ou du Département de l'instruction publique.

Le dernier catalogue étudié dans ce chapitre, daté de 1879 , est celui de la maison Beauchemin, maison qui «était encore active dans le milieu de l'éducation et du commerce du livre scolaire il y a quelques années (p. 212). » Or, la principale caractéristique de ce catalogue, c'est l'émergence de la littérature canadienne, liée elle-même aux politiques de distribution des prix de fin d'année dans les écoles et qui favorisent la littérature « d'ici ». Pas moins de 110 titres « canadiens » figurent ainsi dans une section spéciale du catalogue intitulée «Publications canadiennes et ouvrages sur le Canada. » On ne sera guère surpris d'apprendre que le roman continue son irrésistible ascension avec $46 \%$ de tous les titres littéraires, ceux-ci ayant eux-mêmes progressé de $163 \%$ par rapport au catalogue de 1854 !

L'auteure de l'article procède ensuite à une analyse des genres sur laquelle nous ne nous étendrons pas faute d'espace, préférant en livrer les principales conclusions : (a) «la littérature représente en moyenne, de 1816 à 1879, $28 \%$ du total des titres et $30 \%$ du total des volumes comptabilisés dans les cinq catalogues (p. 226). »; (b) les Québécois ont accès à une partie de la littérature romantique, la plus conventionnelle, semble-t-il, mais la littérature réaliste et surtout naturaliste manquent à l'appel, ce qui ne saurait guère nous étonner. Il est amusant de constater que Voltaire - mais il s'agit bien du Voltaire de la Henriade - fait toujours recette, en compagnie de l'indestructible Mme de Genlis et, bien entendu, de Cooper, Scott et Chateaubriand. Peut-être une étude 
ultérieure nous révèlera-t-elle que, malgré l'œil vigilant de la censure ecclésiastique, quelques titres figurant à l'Index ont pu se glisser çà et là.

Le dernier chapitre de ce passionnant et très riche ouvrage est consacré à "L'offre de titres littéraires dans les catalogues de bibliothèques de collectivités de Montréal (1797-1898) » que traite Isabelle Ducharme. Au total, dix catalogues de bibliothèques de collectivités sont étudiés, soit : (a) trois catalogues $(1797,1824$ et 1833) de la Montreal Library ; (b) le catalogue de 1835 de la Chambre d'Assemblée; (c) le catalogue de 1845 de l'Euvre des bons livres ; (d) les catalogues de 1852, 1870 et le supplément de 1876 de l'Institut canadien ; (e) le catalogue du Collège Sainte-Marie de 1894 ; (f) celui de 1898 du Cercle Ville-Marie.

La Montreal Library / Bibliothèque de Montréal, fondée en 1796, subsistera jusqu'en 1843. Pourvue au départ de titres français, elle verra la proportion de ces derniers diminuer avec le temps, pour disparaitre complètement en 1833, les auteurs français étant désormais disponibles en traduction anglaise. Il s'agit d'une bibliothèque de divertissement, comprenant beaucoup d'œuvres complètes d'auteurs de renom, la proportion des ouvrages littéraires se chiffrant à $42 \%$ en 1833. Même si les auteurs anciens et les classiques affichent leur présence, la bibliothèque - fonctionnant à la manière d'un club fermé - met surtout l'accent sur la littérature contemporaine, répondant par là aux goûts exprimés par une clientèle composée de souscripteurs issus de la bourgeoisie locale anglophone et francophone.

La bibliothèque de la Chambre d'Assemblée, située à Québec, fondée en 1802, sera ouverte au public à compter de 1825. Bibliothèque de travail pour les parlementaires, elle laisse peu de place à la littérature, garnie avant tout d'ouvrages de référence en droit et en politique et d'ouvrages de rhétorique. 
Néanmoins, on y retrouve quelques œuvres d'auteurs anciens et modernes, tels Montaigne, Montesquieu, Chateaubriand et Voltaire. Par contre, les journaux abondent, comme il se doit pour une bibliothèque de ce genre, ainsi que les dictionnaires de langue. Notons qu'Étienne Parent en fut le bibliothécaire.

L'CEuvre des bons livres, créée par les Sulpiciens en 1844, devient le Cabinet de lecture paroissial en 1857, et enfin le Cercle Ville-Marie en 1885. Comme son nom l'indique, son objectif est de contrer les ouvrages jugés « impies », ceux en particulier qui garnissent les rayons de l'Institut canadien. Il importe de signaler que l'Euvre des bons livres donnera naissance à maintes bibliothèques paroissiales qui connaîtront un très grand succès auprès de la population francophone de Montréal, contribuant ainsi de manière non négligeable à sa scolarisation. Quant au Cercle Ville-Marie, il s'adresse, lui, à une clientèle beaucoup plus homogène, socialement parlant, francophone elle aussi, issue majoritairement des milieux étudiants en droit et en médecine, donc bourgeoise.

Le catalogue de 1845 de l'CEuvre des bons livres révèle un pourcentage de $49 \%$ de livres religieux. Quant au catalogue de 1898 du Cercle Ville-Marie, il révèle la présence de $44,8 \%$ de titres littéraires, pour beaucoup des romans édifiants, dont d'ailleurs une bonne proportion de littérature féminine - $40 \%$ de la littérature totale - et une majorité d'auteurs de XIX , dont des auteurs d'ici, notamment Laurier, Parent et Cartier pour l'éloquence, et Fréchette, Lemay, Chapman pour la poésie. On y remarque un intérêt accru pour l'histoire et la critique littéraires, avec Lanson, Lemaître et Villemain. Enfin, le nombre d'œuvres complètes passe de 25 en 1845 à 77 en 1898, et regroupe les grands noms tels Balzac, Musset, Pascal, Homère, Sophocle, Rousseau, sans oublier Casgrain et Crémazie! 
L'Institut canadien de Montréal, dont le clergé finira par avoir raison après la décision de Rome en 1869 d'interdire aux catholiques d'en fréquenter la bibliothèque, et qui fermera ses portes en 1880 dans l'indifférence générale — du côté francophone tout au moins, puisque c'est l'Institut Fraser qui en rachètera le fonds - constitue une bibliothèque unique à Montréal et au Québec au XIX ${ }^{e}$ puisque son objectif avoué était de donner à l'élite bourgeoise et intellectuelle francophone un lieu de rassemblement et d'étude pour tous ceux qui voulaient échapper à la tutelle ecclésiastique et aborder les grands problèmes de l'heure dans une perspective de libre-pensée. C'est d'ailleurs la raison pour laquelle on y retrouve maints titres inscrits à l'Index. Elle ne manque pourtant pas d'auteurs déjà cités dans les bibliothèques contrôlées par le clergé, mais pas nécessairement les mêmes titres, $L a$ Henriade, par exemple, cédant probablement le pas à Candide et au Dictionnaire philosophique! Des auteurs québécois, on retiendra les noms de Gérin-Lajoie, Napoléon Bourassa, Edmond Lareau et James Huston. 1870 marque, semble-t-il, un tournant dans la composition de la bibliothèque, l'accent étant dorénavant davantage mis sur le divertissement que sur la littérature d'idées.

La dernière partie de ce dernier chapitre est consacrée à la bibliothèque du Collège Sainte-Marie. Le catalogue de 1894 révèle, sans surprise, que la majorité des 1925 titres ont été choisis pour répondre aux besoins d'une jeunesse catholique, avec prépondérance accordée aux ouvrages du XIX . Sans surprise, là encore, on y trouve une majorité de titres français, dont sont exclus les habituels trublions : Zola, Sand, Baudelaire, Verlaine, bref tous ces auteurs qui sentent le soufre. Comme il se doit dans un établissement d'enseignement, abondent les traités de rhétorique, d'éloquence et d'exercices littéraires. Quant aux ouvrages québécois, 113 titres y figurent 
avec en tête le roman, suivi de la poésie. Ici encore, l'abbé Casgrain et Crémazie se retrouvent en édition complète. Comme le signale Isabelle Ducharme, la bibliothèque contient « une littérature judicieusement choisie en fonction d'un programme d'enseignement et de lecture rigoureusement prédéterminé (p. 264).»

En conclusion à ce chapitre, quelques remarques s'imposent :(a) le roman est au cœur des collections littéraires, avec prépondérance du roman du XIX ${ }^{e}$, et un roman plus souvent qu'autrement de facture conventionnelle, pour ne pas dire d'édification; (b) le Moyen-Âge et la Renaissance brillent par leur absence, ce qui ne surprend guère, la langue de cette période étant peu accessible à un néophyte; (c) le XVII , le grand siècle classique prend une place importante, de même que le XVIII', encore que ce dernier n'est probablement représenté dans les bibliothèques francophones - à l'exception de celle de l'Institut canadien, bien évidemment - que par les œuvres les moins controversées au plan idéologique. La censure qui s'exerça tout au long du siècle, et tout particulièrement à partir des années 1850, a pour résultat que nombre d'auteurs présents dans les catalogues de cette époque sont aujourd'hui totalement oubliés. Il est vrai que sans censure, bien des auteurs qui figurent dans les catalogues de notre époque seront aussi tombés dans les oubliettes de l'Histoire dans un siècle, ou moins...

Dans leur conclusion au présent ouvrage, Yvan Lamonde et Sophie Montreuil rappellent la distinction qu'il y a lieu d'établir entre le catalogue de la lecture personnelle, et celui de la lecture collective. Dans le premier cas, c'est le lecteur qui est le point de départ, un lecteur qui est autant individuel que social, lecteur privilégié dans la mesure où il projette lui aussi d'écrire, lecteur particulièrement actif donc. Cette découverte du lecteur dans son intimité nous le montre lisant à voix basse, 
ou au contraire à voix haute, quand la lecture se fait, le plus souvent, en famille. Le lecteur privilégié s'approvisionne plus rarement dans les bibliothèques publiques, achetant les ouvrages le plus souvent dans des encans, ou empruntant à des amis. On notera cependant que toutes les catégories de lecteurs s'intéressent en priorité à la littérature, une littérature dont le champ est d'ailleurs beaucoup plus large que de nos jours, et à la lecture du journal. On le sait, au XIX ${ }^{\mathrm{e}}$ la presse est souvent le véhicule privilégié du roman, roman qui d'ailleurs reçoit la consécration qu'il n'a pas cessé de conserver de nos jours.

Signalons que l'ouvrage contient une riche bibliographie de l'histoire de la lecture qui ravira les chercheurs et les amateurs, car ce livre est susceptible d'intéresser un large public qui trouvera ici un complément d'information aux questions qu'il peut se poser concernant la vie intellectuelle de personnages connus, mais aussi de la foule anonyme qui, hier comme aujourd'hui, cherche dans le livre une source d'évasion ou un complément de culture. En bref, un ouvrage qui apporte un contribution majeure à l'étude du Québec au XIX'.

Francis Parmentier

Gatineau 